\title{
Establishment of patient-derived cancer xenografts in immunodeficient NOG mice
}

\author{
TSUYOSHI CHIJIWA ${ }^{1}$, KENJI KAWAI ${ }^{1}$, AKIRA NOGUCHI ${ }^{2}$, HIDEMITSU SATO $^{3}$, AKIMUNE HAYASHI ${ }^{3}$, \\ HARUHIKO $\mathrm{CHO}^{4}$, MANABU SHIOZAWA ${ }^{4}$, TAKESHI KISHIDA ${ }^{5}$, SOICHIRO MORINAGA ${ }^{4}$, \\ TOMOYUKI YOKOSE $^{2}$, MAKOTO KATAYAMA ${ }^{7}$, NOBUO TAKENAKA ${ }^{7}$, HIROSHI SUEMIZU $^{1}$, \\ ROPPEI YAMADA $^{6}$, YOSHIYASU NAKAMURA ${ }^{6}$, TAKASHI OHTSU $^{6}$, YASUO TAKANO ${ }^{6}$, \\ KOHZOH IMAI $^{6}$, YOHEI MIYAGI ${ }^{6}$ and MASATO NAKAMURA ${ }^{1,8}$ \\ ${ }^{1}$ Central Institute for Experimental Animals, Tonomachi, Kawasaki-ku, Kawasaki, Kanagawa 210-0821; \\ Departments of ${ }^{2}$ Pathology, ${ }^{3}$ Neurosurgery, ${ }^{4}$ Gastrointestinal Surgery, ${ }^{5}$ Urology, and ${ }^{6}$ Research Institute, \\ Kanagawa Cancer Center, Nakao, Asahi-ku, Yokohama 241-8515; ${ }^{7}$ Department of Neurosurgery, \\ Kawasaki Municipal Hospital, Shinkawa-tori, Kawasaki 210-0013; ${ }^{8}$ Department of Regenerative Medicine, \\ Tokai University School of Medicine, Shimokasuya, Isehara, Kanagawa 259-1193, Japan
}

Received January 31, 2015; Accepted March 13, 2015

DOI: 10.3892/ijo.2015.2997

\begin{abstract}
Viable and stable human cancer cell lines and animal models combined with adequate clinical information are essential for future advances in cancer research and patient care. Conventional in vitro cancer cell lines are commonly available; however, they lack detailed information on the patient from which they originate, including disease phenotype and drug sensitivity. Patient-derived xenografts (PDX) with clinical information (so-called 'cancer xenopatients') are a promising advance that may accelerate the development of anticancer therapies. We established 61 PDX lines from 116 surgically removed tumor tissues inoculated subcutaneously into NOG mice (53\% success rate). PDX lines were established from various types of epithelial tumors and also from sarcomas, including gastrointestinal stromal tumors and Ewing/PNET sarcomas. The metastatic tumors yielded PDX lines more effectively $(65 \%)$ than the primary tumors $(27 \%$, $\mathrm{P}<0.001)$. In our PDX models, morphological characteristics, gene expression profiles, and genetic alteration patterns were all well preserved. In eight cases $(7 \%)$, the transplantable xenografts for several generations were composed of large monotonous nonepithelial cells of human origin, revealed to be Epstein-Barr virus infection-associated lymphoproliferative lesions. Despite this, PDX linked with clinical information offer many advantages for preclinical studies investigating
\end{abstract}

Correspondence to: Dr Yohei Miyagi, Kanagawa Cancer Center Research Institute, 2-3-2 Nakao, Asahi-ku, Yokohama 241-8515, Japan E-mail: miyagi@gancen.asahi.yokohama.jp

Key words: patient-derived xenograft, NOG mice, tumor model, cancer xenopatients, anticancer therapies new anticancer drugs. The fast and efficient establishment of individual PDX may also contribute to future personalized anticancer therapies.

\section{Introduction}

Animal models have been used in front-line preclinical studies for predicting efficacy and possible toxicities of anticancer drugs in cancer patients $(1,2)$. Current tumor models used for drug evaluation generally consist of implantation into immunodeficient mice of xenografts generated from well-established human cancer cell lines that have already adapted to in vitro growth. These models have been used extensively for decades for rapid screening of anticancer drug efficacy $(3,4)$.

In recent years, xenografts derived from engrafting fresh surgical specimens directly into immunodeficient mice have enabled the development of more relevant in vivo models for human tumors (5). Such patient-derived xenograft (PDX) models, established by direct transfer of tumor tissue, retain similar morphology, architecture, and molecular signatures as the original cancers and thus should be used for rapid screening of potential therapeutics $(6,7)$. Whereas the conventional xenograft models using cell lines provide only a monoclonal mass of tumor cells, PDX models recapitulate not only interactions from the host microenvironments but also the cancerous heterogeneity including the cancer stem cells $(5,6,8)$. Results from these investigations support the use of direct transfer xenografts as a reliable strategy to anticipate clinical findings, provide direction for optimizing personalized treatment in advanced cancers, and suggest novel treatment opportunities in patients with no other therapeutic options (9). The advantages of PDX models in preserving cancer stem cells and the clinical information of the donor patient (so-called 'cancer xenopatient') may allow for accelerated cancer research by simulating the situation in cancer patients more closely $(6,7)$. 
However, the establishment of direct xenografts is still technically difficult $(1,10,11)$. Recently, a new immunodeficient animal model, NOD/Shi-scid/IL-2R $\gamma^{\text {null }}$ (NOG) mice, derived from the NOD/SCID mouse with a common gamma chain, has been introduced. In addition to lacking functional $\mathrm{T}$ and B lymphocytes, the NOG mouse has multifunctional defects in natural killer cell activity, macrophage function, complement activity, and dendritic cell function (12). NOG mice were reported to be the most appropriate immunodeficient host animal for direct xenografting of fresh tumor tissue (5).

In the present study, we investigated the efficient establishment of PDX using NOG mice with clinical factors of xenotransplantation. We also discuss herein the application of this newly developed system for not only reliable preclinical studies of new anticancer drugs but also personalized anticancer therapies.

\section{Materials and methods}

Tumor tissues for transplantation. The 116 surgically removed fresh tumor tissues for transplantation were obtained at Kanagawa Cancer Center (Yokohama, Kanagawa, Japan) and Kawasaki Municipal Hospital (Kawasaki, Kanagawa, Japan) with the patients' written informed consent for the study. The study was performed in collaboration with Keihin Coastal Area Life Innovation Comprehensive Special Zones for International Competitiveness Development (Japan) from 2011 to 2012. The ethics committees independently approved the study (authorization number: 176 at Kanagawa Cancer Center, 23-410 at Kawasaki Municipal Hospital). The entire list of engrafted tumors with the patient profiles is shown in Table I.

Animals. NOG mice, between 6 and 12 weeks of age, were used in this study. The mice were obtained from the Central Institute for Experimental Animals (CIEA; Kanagawa, Japan) (12). All animals were housed in plastic cages $(136 \times 208 \times 115 \mathrm{~mm})$ within a vinyl isolator system $(1150 \times 500 \times 500 \mathrm{~mm})$ in a pathogen-free state, at a temperature of $22 \pm 1^{\circ} \mathrm{C}$ with $45 \pm 10 \%$ humidity, and a $12 \mathrm{~h}$ light $/ 12 \mathrm{~h}$ dark cycle. All experiments involving laboratory animals were performed in accordance with the care and use guidelines of the CIEA, according to our previous studies (13-15). These guidelines meet the generally accepted international criteria on humane treatment that spare the animal needless pain and suffering, and require confirmation that the experiments conducted are of actual scientific benefit to humankind.

Procedures for the establishment of PDX models by serial engraftment. Fresh tumor tissues were divided into three pieces under sterile conditions. One piece of each tissue specimen was immediately placed in Dulbecco's modified minimal essential medium without antibiotics and without fetal bovine serum, and stored at $4^{\circ} \mathrm{C}$ until engrafting. Another piece was cryopreserved for molecular biological examination, and the last piece was fixed in $4 \%$ formaldehyde for histopathological examination. The piece for engraftment was further divided into small pieces $\left(\sim 8-64 \mathrm{~mm}^{3}\right)$ using sterilized surgical scissors. A small incision was made in the leg of each mouse and a transplant needle was inserted until the tip reached the dorsal subcutaneous area. Approximately 10 pieces of tumor tissue were inoculated into the dorsal subcutis via the needle. After the engrafted mass expanded to over quadruple its size, the xenograft tumor was harvested and directly re-transplanted for expansion in later serial generations using the same procedure. After the tumor tissue had been passaged three times or more and histopathological examination confirmed the PDX to be a growing human tumor, we considered the PDX line as 'established'. The established PDX tissue was divided into small pieces, completely submerged in cryopreservation medium (Cellbanker ${ }^{\circledR} 1$, Zenoaq, Fukushima, Japan), and then stored in liquid nitrogen. The frozen tissues were later thawed and used for experiments including re-transplantation and expansion. Mice that did not develop tumor mass over six months after engraftment were sacrificed as 'failed', and this was confirmed histopathologically.

Morphological examination of the primary engrafts and the $P D X$ descendants. For morphological analyses, sample tissues were formalin-fixed, paraffin-embedded (FFPE), sliced into $4-\mu \mathrm{m}$ sections, and subjected to standard hematoxylin and eosin (H\&E) staining or immunohistochemistry (IHC). IHC was performed using the Bond Polymer Refine Detection system (Leica Microsystems, Tokyo, Japan) according to the manufacturer's instructions. Nuclei were counterstained with hematoxylin. Primary antibodies used for IHC were: monoclonal anti-HLA class 1-A, B, C (Hokudo, Sapporo, Japan), rabbit polyclonal anti-c-kit (Nichirei Biosciences, Tokyo, Japan); monoclonal anti-CD34, clone NU-4A1 (Nichirei Biosciences); monoclonal antileukocyte common antigen, clone PD7/26, 2B11 (CD111, Nichirei Biosciences); HER2 (Hercep Test ${ }^{\mathrm{TM}}$, Dako, Japan), monoclonal antiestrogen receptor (ER), clone 1D5 (Nichirei Biosciences); and monoclonal antiprogesterone receptor (PgR), clone A9621 A (Nichirei Biosciences). Chromogenic in situ hybridization (ISH) for Epstein-Barr virus (EBV)-encoded RNA (EBER) was performed using the EBER 1 DNP probe (Ventana/Roche, Tuscon, AZ, USA) and the ISH iView blue plus detection kit (Ventana/Roche) according to the provider's instructions.

Genetic examination of xenograft tumors in NOG mice. The exon 11 deletion mutation in the KIT gene in the 3rd generation xenograft of the gastrointestinal stromal tumor (GIST) was investigated as previously described (16). Briefly, DNA was extracted from the FFPE thin sections of the xenograft tumor and amplified by polymerase chain reaction (PCR) with primers: 5'-gactgagacaataattattaaaag-3' (forward) and 5'-acccaaaaaggtgacatggaaagc-3' (reverse). PCR products were then directly sequenced using the PCR primers and the Sanger's method with Genetic Analyzer 3100 (Applied Biosystems/ Hitachi, Japan). For EWS-FLI1 fusion mRNA detection, total RNA was extracted from the 3rd generation xenograft of the Ewing sarcoma/primitive neuroectodermal tumor (PNET), reverse transcribed to cDNA, and PCR-amplified with primers: EWS-exon 8 (5'-tcctacagccaagctccaagtc-3') and the FLI1 exon 9 (5'-gtgatacagctggcgttggc-3'). The obtained product was directly sequenced as described for the KIT analysis.

Statistical analysis. Statistical comparisons of data sets were performed by a two-sample t-test. The Chi-square test or 
Table I. The entire list of patients from which the engrafted tumors were taken and the fate of the xenografts.

\begin{tabular}{|c|c|c|c|c|c|c|c|}
\hline No. & Age & Gender & Original tumor site & Pathology & Primary/Metastasis & Tumor type & Result \\
\hline 1 & 43 & M & Lung & Adenosquamous carcinoma & Brain metastasis & Epithelial & Established \\
\hline 2 & 60 & M & Lung & Adenocarcinoma & Brain metastasis & Epithelial & Established \\
\hline 3 & 69 & M & Lung & Adenocarcinoma & Brain metastasis & Epithelial & Failed $^{\mathrm{c}}$ \\
\hline 4 & 35 & $\mathrm{~F}$ & Large intestine & Tubular adenocarcinoma & Liver metastasis & Epithelial & Established \\
\hline 5 & 51 & $\mathrm{~F}$ & Breast & Ductal carcinoma & Brain metastasis & Epithelial & Established \\
\hline 6 & 62 & M & Prostate & Adenocarcinoma & Primary & Epithelial & Failed \\
\hline 7 & 65 & M & Large intestine & Tubular adenocarcinoma & Liver metastasis & Epithelial & Established \\
\hline 8 & 76 & $\mathrm{~F}$ & Large intestine & Tubular adenocarcinoma & Liver metastasis & Epithelial & Established \\
\hline 9 & 60 & $\mathrm{~F}$ & Lung & Adenocarcinoma & Brain metastasis & Epithelial & Failed $^{\mathrm{c}}$ \\
\hline 10 & 66 & $\mathrm{~F}$ & Lung & Adenocarcinoma & Brain metastasis & Epithelial & Established \\
\hline 11 & 74 & $\mathrm{~F}$ & Large intestine & Tubular adenocarcinoma & Liver metastasis & Epithelial & Established \\
\hline 12 & 58 & M & Nerve & MPNST & Primary & Mesenchymal & Established \\
\hline 13 & 28 & M & Bone & Ewing/PNET & Brain metastasis & Mesenchymal & Established \\
\hline 14 & 58 & M & Thyroid & Papillary carcinoma & Brain metastasis & Epithelial & Failed \\
\hline 15 & 76 & M & Large intestine & Tubular adenocarcinoma & Liver metastasis & Epithelial & Failed $^{c}$ \\
\hline 16 & 65 & $\mathrm{~F}$ & Breast & Ductal carcinoma & Brain metastasis & Epithelial & Failed \\
\hline 17 & 69 & $\mathrm{~F}$ & Large intestine & Tubular adenocarcinoma & Brain metastasis & Epithelial & Established \\
\hline 18 & 71 & $\mathrm{~F}$ & Large intestine & Tubular adenocarcinoma & Liver metastasis & Epithelial & Established \\
\hline 19 & 74 & M & Esophagus & Squamous cell carcinoma & Brain metastasis & Epithelial & Established \\
\hline 20 & 68 & M & Kidney & Renal cell carcinoma & Brain metastasis & Epithelial & Established \\
\hline 21 & 81 & $\mathrm{~F}$ & Lung & Adenocarcinoma & Brain metastasis & Epithelial & Failed $^{c}$ \\
\hline 22 & 80 & M & Small intestine & GIST & Primary & Mesenchymal & Failed \\
\hline 23 & 55 & M & Prostate & Adenocarcinoma & Primary & Epithelial & Failed \\
\hline 24 & 65 & $\mathrm{~F}$ & Breast & Ductal carcinoma & Brain metastasis & Epithelial & Failed \\
\hline 25 & 51 & M & Large intestine & Tubular adenocarcinoma & Liver metastasis & Epithelial & Established \\
\hline 26 & 66 & M & Pancreas & Ductal carcinoma & Primary & Epithelial & Failed \\
\hline 27 & 40 & $\mathrm{~F}$ & Brain & Glioblastoma & Primary & Mesenchymal & Failed \\
\hline 28 & 61 & M & Large intestine & Tubular adenocarcinoma & Liver metastasis & Epithelial & Established \\
\hline 29 & 43 & M & Brain & Glioblastoma & Primary & Mesenchymal & Failed \\
\hline 30 & 60 & M & Stomach & GIST & Peritoneal metastasis & Mesenchymal & Established \\
\hline 31 & 77 & M & Stomach & Tubular adenocarcinoma & Peritoneal metastasis & Epithelial & Established \\
\hline 32 & 46 & M & Brain & Astrocytoma & Primary & Mesenchymal & Failed \\
\hline 33 & 61 & $\mathrm{~F}$ & Duodenum & Tubular adenocarcinoma & Primary & Epithelial & Failed \\
\hline 34 & 65 & $\mathrm{~F}$ & Breast & Ductal carcinoma & Brain metastasis & Epithelial & Failed \\
\hline 35 & 64 & M & Large intestine & Tubular adenocarcinoma & Liver metastasis & Epithelial & Established \\
\hline 36 & 69 & $\mathrm{~F}$ & Lung & Squamous cell carcinoma & Brain metastasis & Epithelial & Established \\
\hline 37 & 69 & $\mathrm{~F}$ & Uterus body & Adenocarcinoma & Brain metastasis & Epithelial & Established \\
\hline $38^{\mathrm{a}}$ & 58 & $\mathrm{~F}$ & Large intestine & Tubular adenocarcinoma & Liver metastasis & Epithelial & Established \\
\hline 39 & 70 & $\mathrm{~F}$ & Large intestine & Tubular adenocarcinoma & Brain metastasis & Epithelial & Established \\
\hline 40 & 47 & M & Primary unknown & Adenocarcinoma & Brain metastasis & Epithelial & Established \\
\hline 41 & 71 & $\mathrm{~F}$ & Large intestine & Tubular adenocarcinoma & Liver metastasis & Epithelial & Established \\
\hline 42 & 71 & $\mathrm{~F}$ & Uterus body & Adenocarcinoma & Brain metastasis & Epithelial & Failed $^{\mathrm{c}}$ \\
\hline 43 & 55 & $\mathrm{~F}$ & Large intestine & Tubular adenocarcinoma & Liver metastasis & Epithelial & Established \\
\hline 44 & 52 & M & Lung & Small cell carcinoma & Brain metastasis & Epithelial & Established \\
\hline 45 & 68 & M & Prostate & Adenocarcinoma & Primary & Epithelial & Failed \\
\hline 46 & 73 & M & Lung & Adenocarcinoma & Brain metastasis & Epithelial & Failed \\
\hline 47 & 73 & M & Large intestine & Tubular adenocarcinoma & Liver metastasis & Epithelial & Established \\
\hline 48 & 55 & $\mathrm{~F}$ & Breast & Ductal carcinoma & Brain metastasis & Epithelial & Failed \\
\hline 49 & 68 & M & Stomach & Tubular adenocarcinoma & Brain metastasis & Epithelial & Failed $^{\mathrm{c}}$ \\
\hline 50 & 52 & M & Large intestine & GIST & Primary & Mesenchymal & Failed \\
\hline
\end{tabular}


Table I. Continued.

\begin{tabular}{|c|c|c|c|c|c|c|c|}
\hline No. & Age & Gender & Original tumor site & Pathology & Primary/Metastasis & Tumor type & Result \\
\hline 51 & 53 & $\mathrm{~F}$ & Nerve & MPNST & Primary & Mesenchymal & Failed \\
\hline 52 & 64 & $\mathrm{M}$ & Large intestine & Tubular adenocarcinoma & Brain metastasis & Epithelial & Failed \\
\hline 53 & 62 & $\mathrm{~F}$ & Pancreas & Ductal carcinoma & Primary & Epithelial & Established \\
\hline $54^{\mathrm{b}}$ & 70 & M & Pancreas & Ductal carcinoma & Lymph node metastasis & Epithelial & Failed \\
\hline $55^{\mathrm{b}}$ & 70 & M & Pancreas & Ductal carcinoma & Lymph node metastasis & Epithelial & Established \\
\hline $56^{\mathrm{b}}$ & 70 & M & Pancreas & Ductal carcinoma & Lymph node metastasis & Epithelial & Established \\
\hline 57 & 35 & $\mathrm{~F}$ & Stomach & GIST & Primary & Mesenchymal & Failed \\
\hline 58 & 64 & M & Lung & Large cell carcinoma & Brain metastasis & Epithelial & Established \\
\hline 59 & 74 & $\mathrm{~F}$ & Pancreas & Anaplastic carcinoma & Primary & Epithelial & Failed \\
\hline 60 & 71 & $\mathrm{~F}$ & Pancreas & Ductal carcinoma & Primary & Epithelial & Established \\
\hline 61 & 74 & $\mathrm{~F}$ & Pancreas & Ductal carcinoma & Primary & Epithelial & Failed \\
\hline 62 & 70 & M & Kidney & Transitional cell carcinoma & Brain metastasis & Epithelial & Failed \\
\hline 63 & 53 & $\mathrm{~F}$ & Large intestine & Tubular adenocarcinoma & Liver metastasis & Epithelial & Failed \\
\hline 64 & 85 & $\mathrm{~F}$ & Stomach & GIST & Primary & Mesenchymal & Failed \\
\hline 65 & 67 & M & Kidney & Renal cell carcinoma & Brain metastasis & Epithelial & Established \\
\hline 66 & 82 & $\mathrm{M}$ & Lung & Adenocarcinoma & Brain metastasis & Epithelial & Established \\
\hline 67 & 61 & M & Kidney & Renal cell carcinoma & Peritoneal metastasis & Epithelial & Established \\
\hline 68 & 70 & $\mathrm{~F}$ & Stomach & GIST & Primary & Mesenchymal & Failed \\
\hline 69 & 64 & $\mathrm{~F}$ & Brain & Glioblastoma & Primary & Mesenchymal & Failed \\
\hline 70 & 49 & $\mathrm{M}$ & Pancreas & Ductal carcinoma & Primary & Epithelial & Failed \\
\hline 71 & 61 & $\mathrm{~F}$ & Lung & Adenocarcinoma & Brain metastasis & Epithelial & Established \\
\hline 72 & 70 & $\mathrm{~F}$ & Large intestine & Tubular adenocarcinoma & Liver metastasis & Epithelial & Failed $^{c}$ \\
\hline 73 & 72 & $\mathrm{~F}$ & Large intestine & GIST & Primary & Mesenchymal & Failed \\
\hline 74 & 69 & $\mathrm{~F}$ & Stomach & GIST & Primary & Mesenchymal & Failed \\
\hline 75 & 67 & $\mathrm{~F}$ & Large intestine & Tubular adenocarcinoma & Liver metastasis & Epithelial & Established \\
\hline 76 & 79 & M & Brain & Glioblastoma & Primary & Mesenchymal & Failed \\
\hline 77 & 60 & $\mathrm{M}$ & Large intestine & Tubular adenocarcinoma & Liver metastasis & Epithelial & Established \\
\hline 78 & 63 & $\mathrm{~F}$ & Gallbladder & Pleomorphic carcinoma & Brain metastasis & Epithelial & Failed $^{c}$ \\
\hline 79 & 37 & $\mathrm{~F}$ & Large intestine & Tubular adenocarcinoma & Liver metastasis & Epithelial & Failed \\
\hline 80 & 70 & M & Large intestine & Tubular adenocarcinoma & Liver metastasis & Epithelial & Established \\
\hline 81 & 68 & $\mathrm{~F}$ & Stomach & GIST & Primary & Mesenchymal & Failed \\
\hline 82 & 63 & M & Large intestine & Tubular adenocarcinoma & Liver metastasis & Epithelial & Failed \\
\hline 83 & 70 & $\mathrm{~F}$ & Large intestine & Tubular adenocarcinoma & Liver metastasis & Epithelial & Failed \\
\hline 84 & 60 & $\mathrm{~F}$ & Large intestine & Tubular adenocarcinoma & Liver metastasis & Epithelial & Failed \\
\hline 85 & 56 & $\mathrm{M}$ & Large intestine & Tubular adenocarcinoma & Liver metastasis & Epithelial & Established \\
\hline 86 & 58 & $\mathrm{~F}$ & Breast & Ductal carcinoma & Brain metastasis & Epithelial & Established \\
\hline 87 & 16 & $\mathrm{~F}$ & Stomach & GIST & Primary & Mesenchymal & Failed \\
\hline 88 & 62 & $\mathrm{M}$ & Large intestine & Tubular adenocarcinoma & Liver metastasis & Epithelial & Failed \\
\hline 89 & 71 & $\mathrm{M}$ & Pancreas & Ductal carcinoma & Primary & Epithelial & Established \\
\hline 90 & 65 & $\mathrm{~F}$ & Kidney & Renal cell carcinoma & Skin metastasis & Epithelial & Failed \\
\hline 91 & 51 & $\mathrm{M}$ & Pancreas & Ductal carcinoma & Primary & Epithelial & Failed \\
\hline 92 & 51 & $\mathrm{~F}$ & Large intestine & Tubular adenocarcinoma & Liver metastasis & Epithelial & Failed \\
\hline 93 & 75 & M & Lung & Adenocarcinoma & Brain metastasis & Epithelial & Failed \\
\hline 94 & 80 & M & Pancreas & Ductal carcinoma & Primary & Epithelial & Failed \\
\hline 95 & 77 & M & Kidney & Renal cell carcinoma & Skin metastasis & Epithelial & Established \\
\hline 96 & 68 & M & Large intestine & Tubular adenocarcinoma & Liver metastasis & Epithelial & Established \\
\hline 97 & 63 & $\mathrm{M}$ & Pancreas & Ductal carcinoma & Primary & Epithelial & Failed \\
\hline 98 & 61 & $\mathrm{~F}$ & Pancreas & Ductal carcinoma & Primary & Epithelial & Failed \\
\hline 99 & 67 & $\mathrm{~F}$ & Pancreas & Ductal carcinoma & Primary & Epithelial & Established \\
\hline
\end{tabular}


Table I. Continued.

\begin{tabular}{|c|c|c|c|c|c|c|c|}
\hline No. & Age & Gender & Original tumor site & Pathology & Primary/Metastasis & Tumor type & Result \\
\hline 100 & 61 & M & Lung & Adenocarcinoma & Brain metastasis & Epithelial & Established \\
\hline 101 & 71 & M & Stomach & Tubular adenocarcinoma & Brain metastasis & Epithelial & Established \\
\hline $102^{\mathrm{a}}$ & 59 & $\mathrm{~F}$ & Large intestine & Tubular adenocarcinoma & Liver metastasis & Epithelial & Established \\
\hline 103 & 63 & M & Large intestine & Tubular adenocarcinoma & Liver metastasis & Epithelial & Established \\
\hline 104 & 61 & $\mathrm{~F}$ & Thyroid & Follicular carcinoma & Brain metastasis & Epithelial & Established \\
\hline 105 & 71 & M & Pancreas & Ductal carcinoma & Primary & Epithelial & Established \\
\hline 106 & 46 & M & Large intestine & Mucinous adenocarcinoma & Liver metastasis & Epithelial & Established \\
\hline 107 & 66 & M & Pancreas & Ductal carcinoma & Primary & Epithelial & Established \\
\hline 108 & 80 & $\mathrm{~F}$ & Large intestine & Tubular adenocarcinoma & Liver metastasis & Epithelial & Established \\
\hline 109 & 65 & M & Pancreas & Ductal carcinoma & Primary & Epithelial & Established \\
\hline 110 & 81 & $\mathrm{~F}$ & Thyroid & Anaplastic carcinoma & Primary & Epithelial & Established \\
\hline 111 & 71 & M & Pancreas & Ductal carcinoma & Primary & Epithelial & Established \\
\hline 112 & 54 & $\mathrm{~F}$ & Breast & Ductal carcinoma & Brain metastasis & Epithelial & Failed \\
\hline 113 & 62 & M & Large intestine & Tubular adenocarcinoma & Liver metastasis & Epithelial & Established \\
\hline 114 & 68 & M & Lung & Adenocarcinoma & Brain metastasis & Epithelial & Established \\
\hline 115 & 63 & M & Large intestine & Tubular adenocarcinoma & Brain metastasis & Epithelial & Established \\
\hline 116 & 78 & M & Kidney & Renal cell carcinoma & Brain metastasis & Epithelial & Failed \\
\hline
\end{tabular}

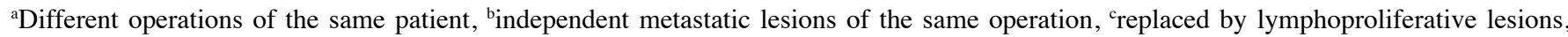
M, male; F, female; MPNST, malignant peripheral nerve sheath tumor; GIST, gastrointestinal stromal tumor.

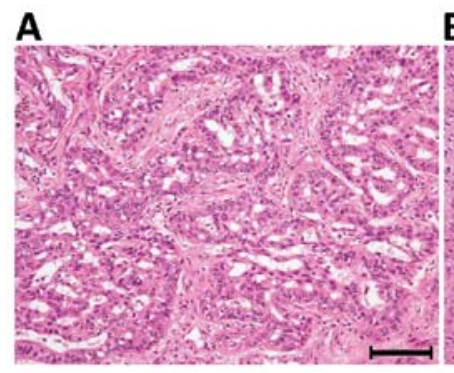

B

C
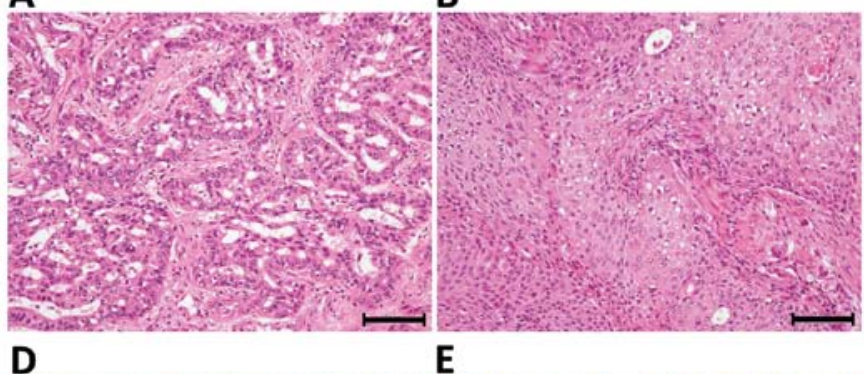

E
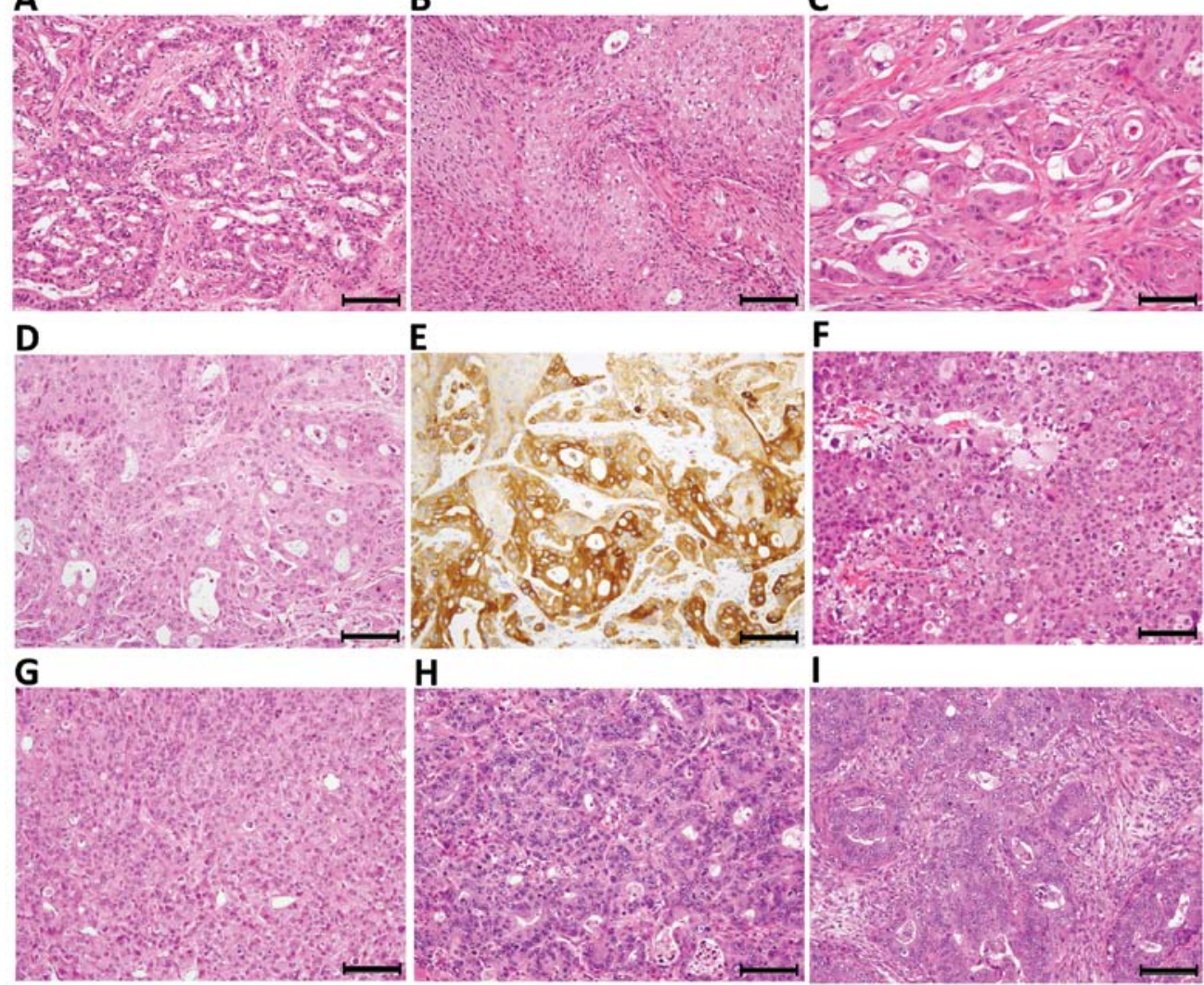

Figure 1. Preserved morphological characteristics observed in the xenograft tumors in NOG mice (A-E) a case of adenosquamous carcinoma of the lung, (F-G) a case of adenocarcinoma of the lung, (H-I) a case of colonic adenocarcinoma. Primary lung carcinoma contained both an adenocarcinoma component (A) and a squamous carcinoma component (B) with few transitional patterns between them. The engrafted brain metastasis showed an admixed histology of adenocarcinoma and squamous carcinoma (C) that was well preserved in the 3rd generation xenograft (D), and immunohistochemically confirmed by the detection of HLA class I (E). The engrafted tumors and the 3rd generation xenograft tumors had similar morphology in NOG PDX lines of a poorly differentiated lung adenocarcinoma ( $\mathrm{F}$ and $\mathrm{G})$ and a moderately differentiated colonic adenocarcinoma $(\mathrm{H}$ and $\mathrm{I}) ; \mathrm{Scale}$ bar, $50 \mu \mathrm{m}$. 
Table II. Summary of the engrafted tumors and the fate of xenografts.

\begin{tabular}{|c|c|c|c|c|c|c|c|}
\hline \multicolumn{2}{|c|}{ Engrafted tumor information } & \multicolumn{2}{|c|}{ Established } & \multicolumn{2}{|c|}{ Failed (LPL) } & \multicolumn{2}{|c|}{ Total } \\
\hline Organ site & Type & Primary & Metastasis & Primary & Metastasis & Primary & Metastasis \\
\hline \multicolumn{8}{|l|}{ Gastrointestinal } \\
\hline Esophagus & Epithelial & 0 & 1 & 0 & 0 & 0 & 1 \\
\hline Stomach & Epithelial & 0 & 2 & 0 & $1(1)$ & 0 & 3 \\
\hline & Mesenchymal & 0 & 1 & 6 & 0 & 6 & 1 \\
\hline Small intestine & Epithelial & 0 & 0 & 1 & 0 & 1 & 0 \\
\hline & Mesenchymal & 0 & 0 & 1 & 0 & 1 & 0 \\
\hline Large intestine & Epithelial & 0 & 25 & 0 & $10(2)$ & 0 & 35 \\
\hline & Mesenchymal & 0 & 0 & 2 & 0 & 2 & 0 \\
\hline \multicolumn{8}{|l|}{ Other digestive } \\
\hline Pancreas & Epithelial & 8 & 2 & 8 & 1 & 16 & 3 \\
\hline Gallbladder & Epithelial & 0 & 0 & 0 & $1(1)$ & 0 & 1 \\
\hline \multicolumn{8}{|l|}{ Respiratory } \\
\hline Lung & Epithelial & 0 & 10 & 0 & $5(3)$ & 0 & 15 \\
\hline \multicolumn{8}{|c|}{ Breast and female genital } \\
\hline Breast & Epithelial & 0 & 2 & 0 & 5 & 0 & 7 \\
\hline Uterus & Epithelial & 0 & 1 & 0 & $1(1)$ & 0 & 2 \\
\hline \multicolumn{8}{|l|}{ Urologic } \\
\hline Kidney & Epithelial & 0 & 4 & 0 & 3 & 0 & 7 \\
\hline \multicolumn{8}{|l|}{ Neurologic } \\
\hline Brain & Mesenchymal & 0 & 0 & 5 & 0 & 5 & 0 \\
\hline Nerve & Mesenchymal & 1 & 0 & 1 & 0 & 2 & 0 \\
\hline \multicolumn{8}{|l|}{ Others } \\
\hline Bone & Mesenchymal & 0 & 1 & 0 & 0 & 0 & 1 \\
\hline Thyroid & Epithelial & 1 & 1 & 0 & 1 & 1 & 2 \\
\hline Prostate & Epithelial & 0 & 0 & 3 & 0 & 3 & 0 \\
\hline Primary unknown & Epithelial & 0 & 1 & 0 & 0 & 0 & 1 \\
\hline
\end{tabular}

LPL, lymphoproliferative lesions.

the two-sided Fisher's probability exact test was applied for comparisons between group frequencies. These analyses were performed using JMP version 11 software (SAS Institute Inc., Cary, NC, USA). P-values of $<0.05$ were considered significant.

\section{Results}

Efficacy of PDX line establishment in NOG mice. In total, 116 surgically removed tumor tissues were engrafted in NOG mice (Tables I and II). The group of patients who provided tumors for this study comprised 63 men and 53 women, with a mean age of 63 years. Thirty-seven tumors were obtained from primary sites and 79 tumors were from metastases. Ninetyeight tumors were epithelial (carcinomas) and 18 tumors were nonepithelial (sarcomas). Tumor specimens were engrafted on the day of surgery or 1-6 days after the surgical removal (owing to sample transport and public holidays). The primary organ site of the transplant together with the difference between the primary tumor or metastasis and the fate (established or failed) are summarized in Table III.

PDX lines were considered established when they were passaged three times or more and histopathological examination confirmed their human origin and their morphological similarity to the corresponding engrafted tumor. Of the 116 tumors engrafted, 61 were established as PDX lines, a success rate of 53\%. On comparing the established cases with the failed cases, no significant differences were observed in age or gender. The average age of patients in established cases was 64 years, compared with 63 years in the failed cases and there was no statistically significant difference $(\mathrm{P}=0.53$, $\mathrm{t}$-test $)$. In the established cases, 38 cases were from male patients $(60 \%)$ and 23 were from female patients $(43 \%)$ cases and there was also no statistically significant difference $(\mathrm{P}=0.09$, Fisher's probability exact test). High establishment rates of PDX lines were observed in tumors of the respiratory system $(67 \%)$, gastrointestinal tumors (58\%), and urological tumors (57\%). None of the 
A

B C
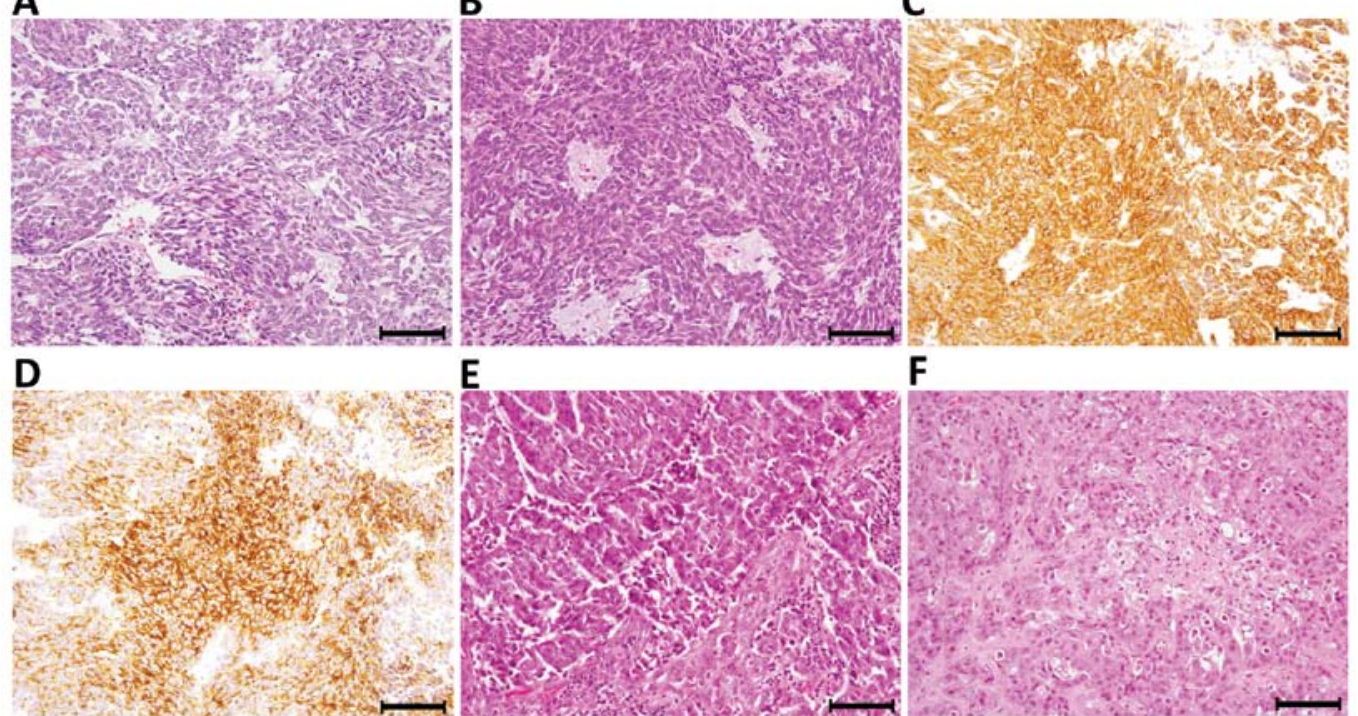

F
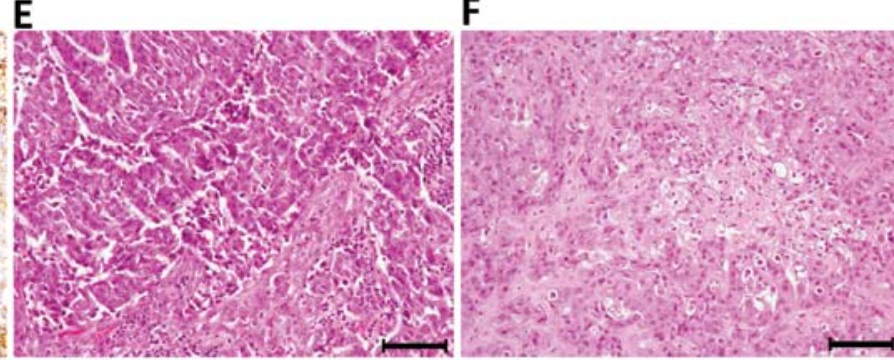

G

H

I
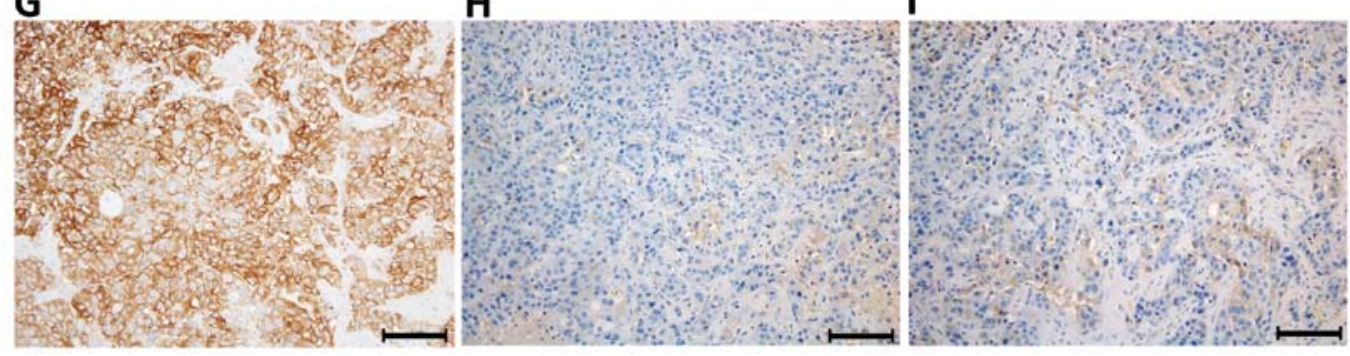

Figure 2. Preserved protein expression evaluated by IHC in the xenograft tumors in NOG mice (A-D) a case of GIST, (E-I) a case of breast cancer. H\&E stained images of the engrafted tumor (A) and the 3rd generation xenograft tumor (B) of the established GIST case are shown. IHC for c-kit (C) and CD34 (D) gave strongly positive signals in the xenograft that reflected those in the engrafted tumor. The 3rd generation xenograft tumor of a HER $23^{+}$breast cancer case showed a similar morphology (F) and similar HER2, ER and PgR IHC patterns (G-I) to the engrafted tumor (E); Scale bar, $50 \mu \mathrm{m}$.

primary prostatic tumors or brain tumors yielded PDX lines in multiple trials.

The establishment rate among the primary organ sites of engrafts was different; however, there was no statistical significance ( $\mathrm{P}=0.29$, Chi-square test). Fifty-eight $\mathrm{PDX}$ lines of carcinomas (59\%) and three of sarcomas (17\%) were established, and the establishment rate was significantly higher for carcinomas $(\mathrm{P}<0.001$, Fisher's probability exact test). Metastatic tumors yielded PDX lines more effectively than tumors from primary sites $(65 \%$ and $27 \%$, respectively; $\mathrm{P}<0.001$, Fisher's probability exact test). Tumors engrafted into NOG mice two or more days after surgical removal showed a higher establishment rate $(61 \%)$ than those engrafted on the day of surgery or the next day (51\%), but there was no statistically significant difference $(\mathrm{P}=0.49$, Fisher's probability exact test).

Preservation of the original tumor characteristics in the PDX of NOG mice. The morphological characteristics of the transplanted tumors, as examined by H\&E-staining, were well maintained in the corresponding xenograft tumors both cellularly and structurally. One representative case of the PDX line derived from a brain metastasis of an adenosquamous carcinoma of the lung is presented in Fig. 1. The primary tumor of the lung consisted of an adenocarcinoma component and a less abundant squamous carcinoma component, and the transitional pattern between them was rare (Fig. 1A and B).
In contrast, the brain metastasis tumor that was engrafted in NOG mice showed a histological structure of an admixture of adenocarcinoma and squamous carcinoma components, and the tumor characteristics were well preserved in the xenograft tumors through the 1st to the 5th generations (Fig. 1C and D). All of the components of the PDX tumor, except for the interstitium, were confirmed as having human origin by IHC for HLA class I (Fig. 1E). Similarly, a brain metastasis of a poorly differentiated adenocarcinoma of the lung was established as a PDX tumor of a poorly differentiated adenocarcinoma (Fig. 1F and $\mathrm{G}$ ), whereas a liver metastasis of a moderately differentiated adenocarcinoma of the colon with a cribriform pattern was established as a PDX tumor with a similar histology, indicating that differentiation capacity was generally preserved (Fig. $1 \mathrm{H}$ and $\mathrm{I}$ ).

Protein expression, as examined by IHC, was also well maintained in PDX tumors. Only one PDX line was successfully established from 10 trials of GIST engraftment (Table I). The established line was derived from a recurrent metastasis after imatinib methylate treatment. The 3rd generation xenograft was examined and revealed to be strongly positive for c-kit (a proto-oncogene) and CD34 (Fig. 2A-D). A PDX line established from a brain metastasis of a HER2-positive breast cancer showed strong membranous positivity $\left(3^{+}\right)$for HER2, and weak dispersed positivity for ER and PgR, and therefore shared the same characteristics as the engrafted tumor (Fig. 2E-I). 

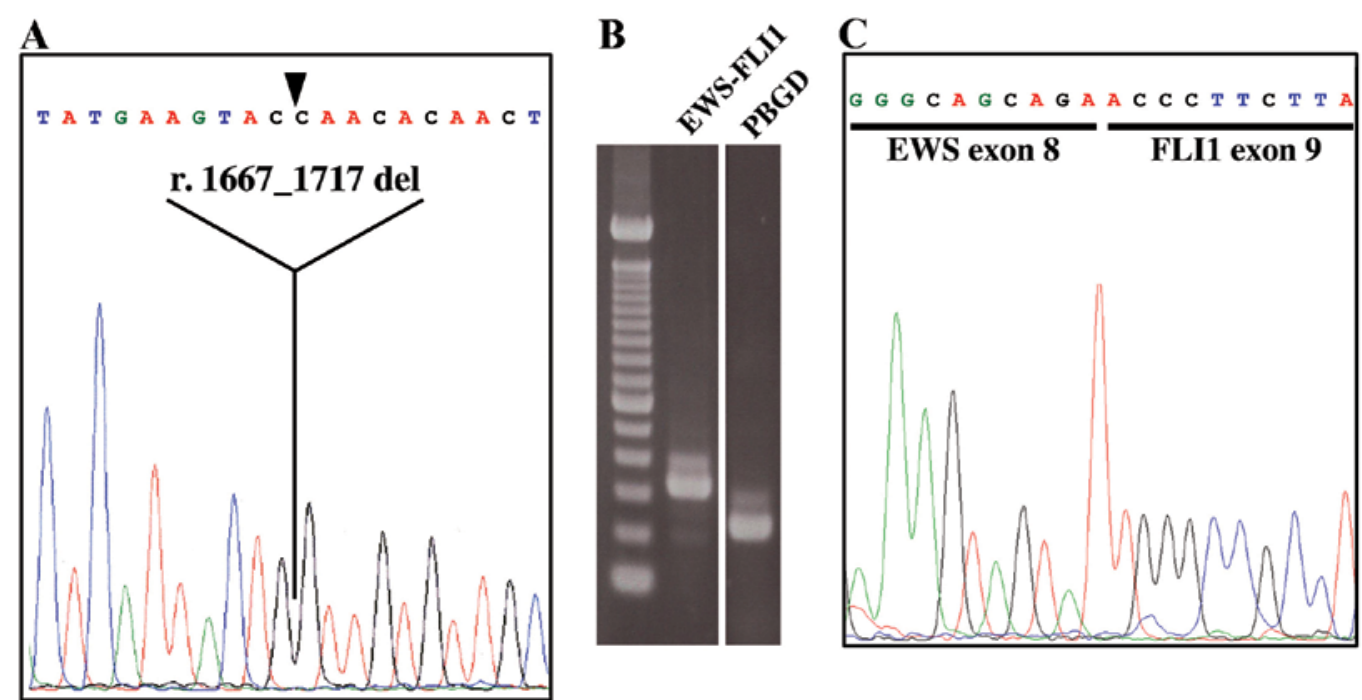

Figure 3. Preserved genetic alterations in the xenograft tumors in NOG mice. (A) Electropherogram of KIT exon 11 nucleotide sequencing for the 3rd generation xenograft tumor. A 51-bp deletion (r.1667_1717 del/p. Q556_D572 del) was detected that was identical to the alteration found in the engrafted tumor. (B) Agarose gel image of the reverse-transcription (RT)-PCR product used to amplify the EWS-FLI1 fusion mRNA. RT-PCR of the PBGD gene, a housekeeping gene, was included as a control. (C) Electropherogram of the EWS-FLI1 fusion RT-PCR product.

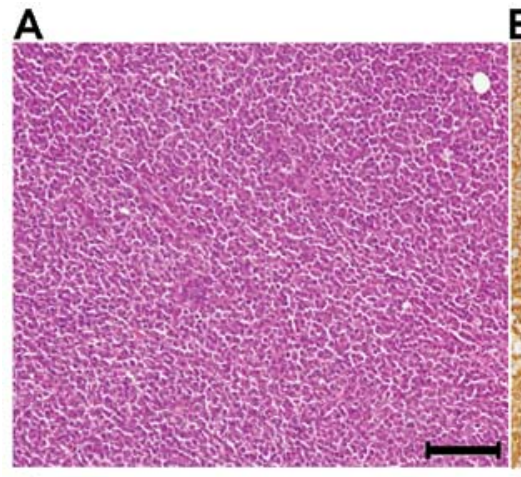

B

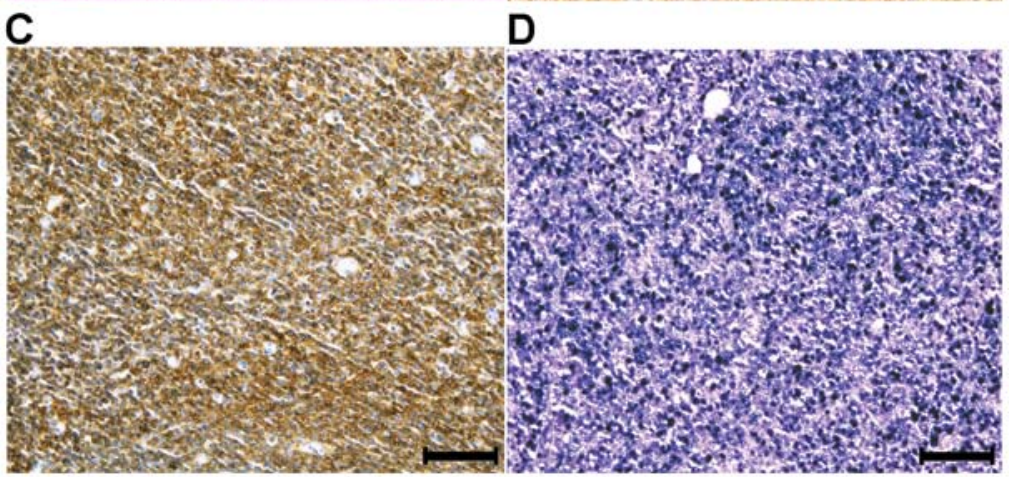

Figure 4. Lymphoproliferative lesion observed in the xenograft tumors. (A) A H\&E-stained image of monotonous nonepithelial cells in a transplantable xenograft tumor; (B) IHC for HLA class 1; (C) IHC for CD111 (leukocyte common antigen); (D) ISH for EBER; Scale bar, $50 \mu \mathrm{m}$.

As expected, genetic alterations were conserved in the PDX tumors of NOG mice. The engrafted GIST tumor contained a 51-nucleotide deletion in exon 11 of KIT (r.1667_1717 del/p. Q556_D572 del) and DNA extracted from the 3rd generation xenograft was found to contain an identical mutation (Fig. 3A). Furthermore, the Ewing/PNET sarcoma xenograft tumor established from a brain metastasis contained the EWS-FLI1 fusion mRNA just as the original engraft (Fig. 3B and C).
Lymphoproliferative lesion ( $L P L)$ in NOG mice. In eight cases (7\% of all engraftments), transplantable xenograft tumors composed of large monotonous nonepithelial cells were observed whose morphology differed from that of the original tumor (Fig. 4A). This phenomenon was observed only in epithelial tumor engraftments (Tables I and II). The monotonous cells were HLA class I positive, demonstrating their human origin, and were also positive for leukocyte common antigen (CD111) by IHC and EBER by ISH, indicating the 
Table III. Comparison of the establishment rate of xenograft lines.

\begin{tabular}{lcrrrr}
\hline & Established & Failed & Total & $\%$ & P-value \\
\hline Original tumor sites & & & & & $0.29^{\mathrm{a}}$ \\
Gastrointestinal & 29 & 21 & 50 & 58 & \\
Other digestive & 10 & 10 & 20 & 50 & \\
Respiratory & 10 & 5 & 15 & 67 & \\
Breast and female & 3 & 6 & 9 & 33 & \\
genital & & & & & \\
Urological & 4 & 3 & 7 & 57 & \\
Neurological & 1 & 6 & 7 & 14 & \\
Others & 4 & 4 & 8 & 50 & \\
Total & 61 & 55 & 116 & 53 & \\
Tumor type & & & & & $<0.001^{\mathrm{b}}$ \\
Carcinomas & 58 & 40 & 98 & 59 & \\
Sarcomas & 3 & 15 & 18 & 17 & \\
Tumor site & & & & & $<0.001^{\mathrm{b}}$ \\
Primary & & & & & \\
Metastasis & 10 & 27 & 37 & 27 & \\
Time to engraftment ${ }^{\mathrm{c}}$ & 51 & 28 & 79 & 65 & \\
Early & & & & & $0.49^{\mathrm{b}}$ \\
Delayed & 47 & 46 & 93 & 51 & \\
\hline
\end{tabular}

${ }^{a}$ Chi-square test, 'btwo-sided Fisher's probability exact test, 'engraftment on the day of surgery or the next day was considered 'early', and engraftment after 2 days was considered as 'delayed'.

possibility that they were EBV infection-associated LPLs (Fig. 4B-D).

\section{Discussion}

In this study, we aimed to establish a PDX line in NOG mice that preserved the original characteristics of the engrafted tumor. In a previous PDX trial in NOG mice, which included more than 300 surgically removed tumors, the establishment rate of the xenograft line was $16 \%$ (41 of 259 engrafts) for primary tumors, $31 \%$ (5 of 16 engrafts) for distant metastasis sites, and 16\% (8 of 51 engrafts) for lymph node metastases (5). In this study, we achieved higher establishment rates both for primary tumors (27\%) and metastatic tumors (65\%). However, the constitution of the tumors used for PDX and those in the primary organ site, the ratios of primary tumors to metastatic tumors, and the numbers of each case all differed between our study and the previous study, making comparisons difficult. For example, the previous study in NOG mice used 57 primary breast cancers and obtained only three PDX lines (5\%), whereas primary tumors of the breast were not included in our study (5). In fact, the establishment rate may depend on the organ site from which the engraft is taken.

Colorectal tumors showed relatively higher establishment rates than tumors from other sites in nude or SCID mice $(10,11)$; this was also the case in the previous NOG study (17/48 engraftments, 35\%) (5). In the present study, establishment rates were found to differ between sites (e.g., 58\% for gastrointestinal tumors compared with $14 \%$ for urological tumors) but this difference was not statistically significant. The establishment rates of metastatic tumors were significantly higher than those of primary tumors, a finding that was consistent with previous studies $(5,17,18)$. In our study, carcinomas showed a significantly higher establishment rate (59\%) than nonepithelial tumors (17\%); however, a limited number of nonepithelial tumors were tested and a larger sample size would be needed to confirm this difference.

In the present study, only subcutaneous transplantations were performed. The transplantation site has been reported to have an influence on xenograft growth (5,19-21). Considering what is known about tumor cells and microenvironmental biology, heterotopic subcutaneous tumor models seem to have some shortcomings compared with orthotopic transplantation, especially in the establishment rate and preservation of the original tumor characteristics (21). However, our subcutaneous models revealed well-preserved characteristics of the original engrafts in morphology, protein expression and gene alterations. Although further studies are needed to clarify whether this was because of the highly immunodeficient nature of NOG mice, the establishment of PDX using manageable subcutaneous transplantations is convenient when compared with skillful orthotopic transplantation.

Unexpectedly, in the present study, we experienced no significant difference in the establishment rate of PDX lines between the tumors engrafted early (on the day of surgery or the next day) and the tumors engrafted after 2 days. To the best of our knowledge, no previous studies have investigated this issue. One could speculate that the so-called cancer stem cells responsible for tumorigenicity in mice might be resistance to the severe stress induced by removal from the patients. Although further investigation is needed, this information might help oncology researchers to improve and simplify PDX line establishment, particularly in light of our findings that subcutaneous transplantation is not inferior to orthotopic transplantation with regard to preserving the original engraft characteristics.

The high occurrence of LPL was the most problematic aspect of the establishment of NOG mice-PDX, which arose because of the severely immunodeficient nature of the animal model. In eight cases (7\% of all engraftments), the engrafted tumors were replaced by LPL until the 3rd generation of xenografts. The LPLs were demonstrated to be EBV infectionassociated, as has been previously reported $(20,22,23)$. Fujii and colleagues also reported that EBV-infected B cells originating from the donor were distributed systemically within the NOG mouse (23). LPLs were transplantable, and difficult to distinguish from the proper xenografts in terms of gross appearance. It is therefore important that histology of xenografts is checked before transplantation into new mice. However, the frequency of LPLs is acceptable when considering the merits of NOG mice. Replacement of the engrafted tumors by LPL accounted for $15 \%$ of the failed cases in PDX line establishment, indicating that the major cause of failed cases is therefore likely to be the nature of the xenografts.

Owing to progression in the field of oncology, the demand for relevant human tumor models is increasing. In vivo models play a vital role in the extrapolation of data to human patients, especially in the development of anticancer agents. Evidence 
that both tumor differentiation and tumor structure were highly conserved between the original surgical specimen and the PDX tumor confirms the suitability of our mouse model for the study of tumor biology. Applications of this model, not only for more common tumors, but also for uncommon tumors, such as sarcomas or pediatric tumors, will provide researchers with reliable comparative preclinical data that may contribute to the development of novel cancer therapies. The rapid and efficient establishment of PDX linking with clinical information may lead in the future to the development of personalized anticancer therapies by simulating various treatments in individual PDX mice, so-called cancer xenopatients.

\section{Acknowledgements}

The authors thank Ms. Aki Namiki, Miyuki Kon, Takayo Shimamura, and Manami Ino at the Kanagawa Cancer Center for their assistance in tissue preparation and obtaining informed consent from patients involved in the study. This work was partly supported by JSPS Grant-in-Aid for Scientific Research (C), numbers 25430098 and 26430097.

\section{References}

1. Morton CL and Houghton PJ: Establishment of human tumor xenografts in immunodeficient mice. Nat Protoc 2: 247-250, 2007.

2. Jin K, Li G, Cui B, Zhang J, Lan H, Han N, Xie B, Cao F, He K, Wang $\mathrm{H}$, et al: Assessment of a novel VEGF targeted agent using patient-derived tumor tissue xenograft models of colon carcinoma with lymphatic and hepatic metastases. PLoS One 6: e28384, 2011.

3. Johnson JI, Decker S, Zaharevitz D, Rubinstein LV, Venditti JM, Schepartz S, Kalyandrug S, Christian M,Arbuck S, Hollingshead M, et al: Relationships between drug activity in NCI preclinical in vitro and in vivo models and early clinical trials. $\mathrm{Br} \mathrm{J}$ Cancer 84 : 1424-1431, 2001.

4. Sausville EA, Burger AM, Becher OJ and Holland EC: Contributions of human tumor xenografts to anticancer drug development. Cancer Res 66: 3351-3354, 2006.

5. Fujii E, Suzuki M, Matsubara K, Watanabe M, Chen YJ, Adachi K, Ohnishi Y, Tanigawa M, Tsuchiya M and Tamaoki N: Establishment and characterization of in vivo human tumor models in the NOD/SCID/gamma(c)(null) mouse. Pathol Int 58: 559-567, 2008.

6. Bertotti A, Migliardi G, Galimi F, Sassi F, Torti D, Isella C, Corà D, Di Nicolantonio F, Buscarino M, Petti C, et al: A molecularly annotated platform of patient-derived xenografts ('xenopatients') identifies HER2 as an effective therapeutic target in cetuximab-resistant colorectal cancer. Cancer Discov 1: 508-523, 2011.

7. Galimi F, Torti D, Sassi F, Isella C, Corà D, Gastaldi S, Ribero D, Muratore A, Massucco P, Siatis D, et al: Genetic and expression analysis of MET, MACC1, and HGF in metastatic colorectal cancer: Response to met inhibition in patient xenografts and pathologic correlations. Clin Cancer Res 17: 3146-3156, 2011.

8. Kobayashi S, Yamada-Okabe H, Suzuki M, Natori O, Kato A, Matsubara K, Jau Chen Y, Yamazaki M, Funahashi S, Yoshida K, et al: LGR5-positive colon cancer stem cells interconvert with drug-resistant LGR5-negative cells and are capable of tumor reconstitution. Stem Cells 30: 2631-2644, 2012.
9. Scott CL, Becker MA, Haluska P and Samimi G: Patient-derived xenograft models to improve targeted therapy in epithelial ovarian cancer treatment. Front Oncol 3: 295, 2013.

10. Tokunaga T, Nakamura M, Oshika Y, Ohnishi Y and Ueyama Y: Is xenotransplantability of human colon cancers in SCID mice affected by angiogenic factors? J Natl Cancer Inst 90: 400-401, 1998.

11. Dangles-Marie V, Pocard M, Richon S, Weiswald LB, Assayag F, Saulnier P, Judde JG, Janneau JL, Auger N, Validire P, et al: Establishment of human colon cancer cell lines from fresh tumors versus xenografts: Comparison of success rate and cell line features. Cancer Res 67: 398-407, 2007.

12. Ito M, Hiramatsu H, Kobayashi K, Suzue K, Kawahata M, Hioki K, Ueyama Y, Koyanagi Y, Sugamura K, Tsuji K, et al: NOD/SCID/gamma(c)(null) mouse: An excellent recipient mouse model for engraftment of human cells. Blood 100: 3175-3182, 2002.

13. Suemizu H, Monnai M, Ohnishi Y, Ito M, Tamaoki N and Nakamura M: Identification of a key molecular regulator of liver metastasis in human pancreatic carcinoma using a novel quantitative model of metastasis in NOD/SCID/gammacnull (NOG) mice. Int J Oncol 31: 741-751, 2007.

14. Chijiwa T, Abe Y, Ikoma N, Yamazaki H, Tsukamoto $H$, Suemizu H, Kawai K, Wakui M, Nishime C, Matsumoto H, et al: Thrombospondin 2 inhibits metastasis of human malignant melanoma through microenvironment-modification in NOD/ SCID/gammaCnull (NOG) mice. Int J Oncol 34: 5-13, 2009.

15. Kubo A, Ohmura M, Wakui M, Harada T, Kajihara S, Ogawa K, Suemizu H, Nakamura M, Setou M and Suematsu M: Semiquantitative analyses of metabolic systems of human colon cancer metastatic xenografts in livers of superimmunodeficient NOG mice. Anal Bioanal Chem 400: 1895-1904, 2011.

16. Cho H, Watanabe T, Aoyama T, Hayashi T, Yamada T, Ogata T, Yoshikawa T, Tsuburaya A, Sekiguchi H, Nakamura Y, et al: Small bud of probable gastrointestinal stromal tumor within a laparoscopically-resected gastric schwannoma. Int J Clin Oncol 17: 294-298, 2012.

17. Yoshida T, Kinoshita H, Segawa T, Nakamura E, Inoue T, Shimizu Y, Kamoto T and Ogawa O: Antiandrogen bicalutamide promotes tumor growth in a novel androgen-dependent prostate cancer xenograft model derived from a bicalutamide-treated patient. Cancer Res 65: 9611-9616, 2005.

18. Marangoni E, Vincent-Salomon A, Auger N, Degeorges A, Assayag F, de Cremoux P, de Plater L, Guyader C, De Pinieux G, Judde JG, et al: A new model of patient tumor-derived breast cancer xenografts for preclinical assays. Clin Cancer Res 13: 3989-3998, 2007

19. Mueller BM and Reisfeld RA: Potential of the scid mouse as a host for human tumors. Cancer Metastasis Rev 10: 193-200, 1991.

20. Bankert RB, Hess SD and Egilmez NK: SCID mouse models to study human cancer pathogenesis and approaches to therapy: Potential, limitations, and future directions. Front Biosci 7: c44-c62, 2002.

21. Bibby MC: Orthotopic models of cancer for preclinical drug evaluation: Advantages and disadvantages. Eur J Cancer 40: 852-857, 2004.

22. Itoh T, Shiota M, Takanashi M, Hojo I, Satoh H, Matsuzawa A, Moriyama T, Watanabe T, Hirai K and Mori S: Engraftment of human non-Hodgkin lymphomas in mice with severe combined immunodeficiency. Cancer 72: 2686-2694, 1993.

23. Fujii E, Kato A, Chen YJ, Matsubara K, Ohnishi Y and Suzuki M: Characterization of EBV-related lymphoproliferative lesions arising in donor lymphocytes of transplanted human tumor tissues in the NOG mouse. Exp Anim 63: 289-296, 2014. 